\title{
Geometrically Frustrated Kagome Lattice with Chemical Disorder
}

\author{
R. Szymczak, M. Baran, J. Fink-Finowicki, \\ B. Krzymanska, P. Aleshrevych and H. Szymczak \\ Institute of Physics, Polish Academy of Sciences \\ al. Lotników 32/46, 02-668 Warsaw, Poland
}

\begin{abstract}
The effect of nonmagnetic $\mathrm{Mg}^{2+}$ and magnetic $\mathrm{Co}^{2+}$ doping in Kagome compounds was investigated using DC magnetic susceptibility. The main effect of doping is to change the crystal field parameters. It was shown that $\mathrm{Co}^{2+}$ doping increases considerably crystal field parameters in $\mathrm{Ni}_{3} \mathrm{~V}_{2} \mathrm{O}_{8}$ while doping with nonmagnetic $\mathrm{Mg}^{2+}$ ions decreases considerably crystal field parameters in $\mathrm{Co}_{3} \mathrm{~V}_{2} \mathrm{O}_{8}$ crystals.
\end{abstract}

PACS numbers: 75.30.Cr, 75.20.--g

\section{Introduction}

One of the most studied geometrically frustrated systems is the twodimensional kagome lattice. Recently, the isostructural compounds $\mathrm{M}_{3} \mathrm{~V}_{2} \mathrm{O}_{8}$ $(\mathrm{M}=\mathrm{Ni}, \mathrm{Co}, \mathrm{Cu}, \mathrm{Mg})$ were shown to be new variants of the kagome lattice [1-11]. Among them the most frequently studied of the $\mathrm{M}_{3} \mathrm{~V}_{2} \mathrm{O}_{8}$ systems have been $\mathrm{Ni}_{3} \mathrm{~V}_{2} \mathrm{O}_{8}$ and $\mathrm{Co}_{3} \mathrm{~V}_{2} \mathrm{O}_{8}$. Despite these materials having identical crystal symmetry and similar structural parameters, their magnetic properties are quite different. The difference between the two materials may, in part, be attributed to the magnetocrystalline anisotropy, which is found to be far larger in $\mathrm{Co}_{3} \mathrm{~V}_{2} \mathrm{O}_{8}$ than in $\mathrm{Ni}_{3} \mathrm{~V}_{2} \mathrm{O}_{8}$ [6]. The magnetic behavior of these materials may be described in terms of two inequivalent magnetic ion sites, known as spine sites and crosstie sites. The ordering in both materials involves spin component aligned along the $a$-direction. The magnetic structure always involves ordering of the spine spins. Magnetic susceptibility, neutron diffraction and specific heat measurements revealed that both $\mathrm{Ni}_{3} \mathrm{~V}_{2} \mathrm{O}_{8}$ and $\mathrm{Co}_{3} \mathrm{~V}_{2} \mathrm{O}_{8}$ undergo a series of magnetic phase transitions versus temperature and magnetic field. Recently, we have studied [12] the phase diagram of cobalt-doped geometrically frustrated $\mathrm{Ni}_{3} \mathrm{~V}_{2} \mathrm{O}_{8}$ single crystals. This paper is a continuation of the studies presented in [12] focused on crystal field effects in pure and doped kagome systems. 


\section{Experimental results and discussion}

The $\mathrm{Ni}_{3-3 x} \mathrm{Mg}_{3 x} \mathrm{~V}_{2} \mathrm{O}_{8}(x=0$ and 0.03$)$ and $\mathrm{Co}_{3-3 x} \mathrm{Mg}_{3 x} \mathrm{~V}_{2} \mathrm{O}_{8}(x=0,0.05$, and 0.1 ) single crystals were grown by a method of spontaneous crystallization. The samples were found to be single phase by X-ray diffraction measurements with the crystal structure of orthorhombic space group $(\mathrm{Cmca})$. Magnetic measurements were performed in temperature range from $2 \mathrm{~K}$ to $300 \mathrm{~K}$ using a commercial SQUID magnetometer (MPMS-5, Quantum Design).

Figures 1 and 2 display two examples of the inverse magnetic susceptibility $\chi^{-1}(T)$ at the magnetic field $H=6 \mathrm{kOe}$ applied along the $a, b$, and $c$-axes.
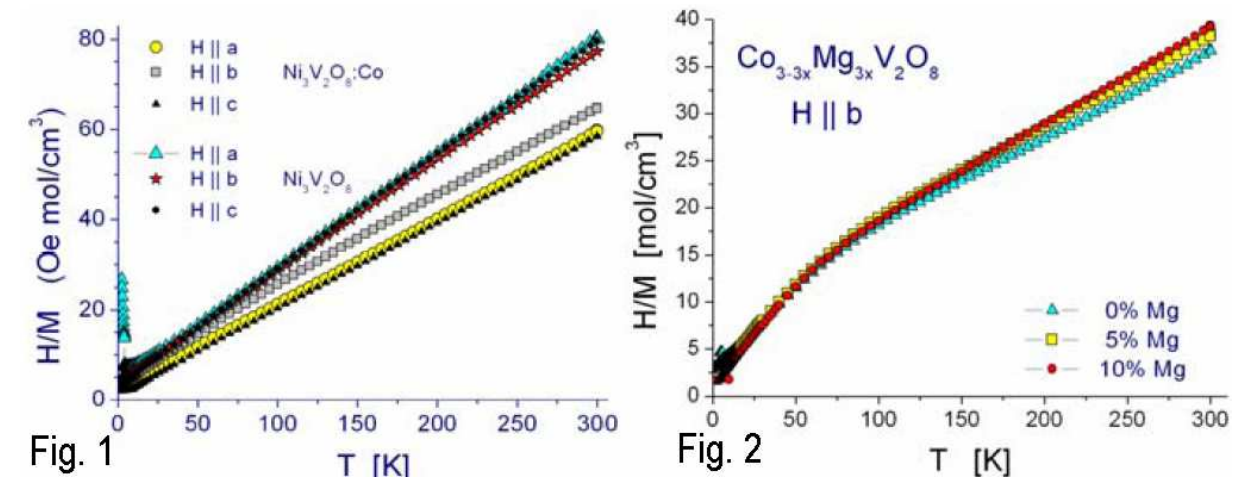

Fig. 1. $\chi^{-1}(T)$ for magnetic field applied along each of the crystallographic directions of $\mathrm{Ni}_{3(1-x)} \mathrm{Co}_{3 x} \mathrm{~V}_{2} \mathrm{O}_{8}(x=0,0.03)$.

Fig. 2. $\chi^{-1}(T)$ for magnetic field applied along the $b$-axis of $\mathrm{Co}_{3(1-x)} \mathrm{Mg}_{3 x} \mathrm{~V}_{2} \mathrm{O}_{8}(x=0$, $0.05,0.1)$.

Important information concerning magnetocrystalline anisotropy and crystal field parameters may be obtained from the high temperature susceptibility tensor, whose components are given asymptotically at high temperatures $\left(T \gg\left|\theta_{i}\right|\right)$ as

$$
\chi_{i i}=C_{i} /\left(T+\theta_{i}\right)
$$

where $i$ labels the Cartesian components in orthorhombic coordinate system. For high temperatures, a fit of the measured susceptibilities to Eq. (1) yields the $\theta_{i}$ values gathered in Table. The experimental data will be analyzed using the Hamiltonian of the form

$$
\mathcal{H}=\sum_{i} H_{\mathrm{cr}}^{i}-\frac{1}{2} \sum_{i \neq j} I_{i j} J_{i} J_{j}+g_{j} \mu_{\mathrm{B}} \boldsymbol{H} \sum_{i} J_{i},
$$

where

$$
H_{\mathrm{cr}}^{i}=\sum_{k, q} \boldsymbol{B}_{k q} \boldsymbol{O}_{k}^{q}
$$

describes the crystal field potential; $\boldsymbol{J}$ is the angular momentum; $I_{i j}$ is the exchange integral; $\mu_{\mathrm{B}}$ is the Bohr magneton, $\boldsymbol{O}_{k}^{q}$ are the Stevens operators. 
Following [13], the Hamiltonian $\mathcal{H}$ may be considered in molecular field approximation which results in the following expressions for crystal field and molecular field parameters and their relation with magnetic susceptibility $\chi_{i i}$ :

$$
\begin{aligned}
& \chi_{i i}^{-1}=3 T /\left[g_{j}^{2} \mu_{\mathrm{B}}^{2} J(J+1)\left(1+\theta_{i} / T\right)\right]-\lambda, \\
& \lambda=\operatorname{const}\left(\theta_{x}+\theta_{y}+\theta_{z}\right), \quad \xi B_{20}=(1 / 3)\left(2 \theta_{z}-\theta_{x}-\theta_{y}\right), \\
& \xi B_{22}=\theta_{x}-\theta_{y}, \quad \xi=(1 / 5)(2 J-1)(2 J+3),
\end{aligned}
$$

$\lambda$ is the molecular field parameter proportional to the exchange integral $I_{i j}$. The results of the calculations of $B_{20}, B_{22}$ parameters are presented in Table. It results from the Table that:

TABLE

Paramagnetic Curie-Weiss temperatures and crystal field pa-

\begin{tabular}{|c|c|c|c|c|c|}
\hline Materials & $\begin{array}{c}\mathrm{NiV}_{2} \mathrm{O}_{8} \\
\theta[\mathrm{K}]\end{array}$ & $\begin{array}{c}\mathrm{CoV}_{2} \mathrm{O}_{8} \\
\theta[\mathrm{K}]\end{array}$ & $\begin{array}{c}\mathrm{NiV}_{2} \mathrm{O}_{8} \\
+3 \% \mathrm{Co} \\
\theta[\mathrm{K}] \\
\end{array}$ & $\begin{array}{c}\mathrm{CoV}_{2} \mathrm{O}_{8} \\
+5 \% \mathrm{Mg} \\
\theta[\mathrm{K}]\end{array}$ & $\begin{array}{c}\mathrm{CoV}_{2} \mathrm{O}_{8} \\
+10 \% \mathrm{Mg} \\
\theta[\mathrm{K}]\end{array}$ \\
\hline$H \| a$ & 15.8 & -6.3 & 11.4 & -4.9 & -2.7 \\
\hline$H \| b$ & 17.6 & 114.0 & 31.1 & 99.0 & 86.0 \\
\hline$H \| c$ & 15.2 & 7.2 & 7.1 & 11.9 & 8.3 \\
\hline$\sum \theta_{i}$ & 48.4 & 114.9 & 49.6 & 106.0 & 91.6 \\
\hline$B_{0}^{2}$ & -1.0 & -13.0 & -9.4 & -9.8 & -9.4 \\
\hline$B_{2}^{2}$ & -1.8 & -50.1 & -19.7 & -43.3 & -33.5 \\
\hline
\end{tabular}
rameters for $\mathrm{Ni}_{3-3 x} \mathrm{Mg}_{3 x} \mathrm{~V}_{2} \mathrm{O}_{8} \quad(x=0$ and 0.03$)$ and $\mathrm{Co}_{3-3 x} \mathrm{Mg}_{3 x} \mathrm{~V}_{2} \mathrm{O}_{8}(x=0,0.05$, and 0.1$)$.

- Chemical disorder introduced by low level doping has rather weak effect on exchange interactions in kagome system. This effect is weaker for the Ni doping than for $\mathrm{Mg}$ doping.

- Crystal field is considerably weaker in $\mathrm{Ni}_{3} \mathrm{~V}_{2} \mathrm{O}_{8}$ than in $\mathrm{Co}_{3} \mathrm{~V}_{2} \mathrm{O}_{8}$ crystals. Co doping increases considerably crystal field parameters in $\mathrm{Ni}_{3} \mathrm{~V}_{2} \mathrm{O}_{8}$ and induces an easy axis along $c$-direction in agreement with experimental data presented in [12]. In contrast, doping with nonmagnetic $\mathrm{Mg}^{2+}$ ions decreases considerably crystal field parameters in $\mathrm{Co}_{3} \mathrm{~V}_{2} \mathrm{O}_{8}$ crystals. From the presented results it is seen that the $a$-axis is an easy axis for pure and $\mathrm{Mg}$-doped $\mathrm{Co}_{3} \mathrm{~V}_{2} \mathrm{O}_{8}$ crystals. The specific behavior of $\mathrm{Co}^{2+}$ ions is, without any doubt, related to the Jahn-Teller character of these ions.

\section{Acknowledgments}

The work was supported in part by the MNiSW under the project N202 057 32/1201. A 


\section{References}

[1] N. Rogado, G. Laves, D.A. Huse, A.P. Ramirez, R.J. Cava, Solid State Commun. 124, 229 (2002).

[2] N. Rogado, M.K. Haas, G. Laves, D.A. Huse, A.P. Ramirez, R.J. Cava, J. Phys., Condens. Matter 15, 907 (2003).

[3] G. Balakrishnan, O.A. Petrenko, M.R. Lees, D.M.K. Paul, J. Phys., Condens. Matter 16, L347 (2004).

[4] G. Laves, A.B. Harris, T. Kimura, N. Rogado, R.J. Cava, A. Aharony, O. Entin-Wohlman, T. Yildirim, M. Kenzelmann, C. Broholm, A.P. Ramirez, Phys. Rev. Lett. 95, 097205 (2005).

[5] G. Lawes, M. Kenzelmann, N. Rogado, K.H. Kim, G.A. Jorge, R.J. Cava, A. Aharony, O. Entin-Wohlman, A.B. Harris, T. Yildirim, Q.Z. Huang, S. Park, C. Broholm, A.P. Ramirez, Phys. Rev. Lett. 93, 247201 (2004).

[6] R. Szymczak, M. Baran, R. Diduszko, J. Fink-Finowicki, M. Gutowska, A. Szewczyk, H. Szymczak, Phys. Rev. B 73, 094425 (2006).

[7] M. Kenzelmann, A.B. Harris, A. Aharony, O. Entin- Wohlman, T. Yildirim, Q. Huang, S. Park, G. Lawes, C. Broholm, N. Rogado, R.J. Cava, K.H. Kim, G. Jorge, A.P. Ramirez, Phys. Rev. B 74, 014429 (2006).

[8] Y. Chen, J.W. Lynn, Q. Huang, F.M. Woodward, T. Yildirim, G. Lawes, A.P. Ramirez, N. Rogado, R.J. Cava, A. Aharony, O. Entin-Wohlman, A.B. Harris, Phys. Rev. B 74, 014430 (2006).

[9] N. Qureshi, H. Fuess, H. Ehrenberg, T.C. Hansen, C. Ritter, K. Prokes, A. Podlesnyak, G. Schwabe, Phys. Rev. B 74, 212407 (2006).

[10] A.B. Harris, T. Yildirim, A. Aharony, O. Entin-Wohlman, Phys. Rev. B 73, 184433 (2006)

[11] N. Qureshi, H. Fuess, H. Ehrenberg, T.C. Hansen, G. Schwabe, Solid State Commun. 142, 169 (2007).

[12] H. Szymczak, M. Baran, R. Szymczak, S.N. Barilo, G.L. Bychkov, S.V. Shiryaev, Acta Phys. Pol. A 111, 71 (2007).

[13] A.K. Zvezdin, V.M. Matveev, A.A. Mukhin, A.I. Popov, Rare Earth Ions in Magnetically Ordered Crystals, Nauka, Moscow 1995 (in Russian). 